\title{
RESISTÊNCIA À INSULINA E DIABETES TIPO 2: UMA ANÁLISE TRANSVERSAL EM UM PROGRAMA DE INTERVENÇÃO NUTRICIONAL
}

\author{
INSULIN RESISTANCE AND TYPE 2 DIABETES: A TRANSVERSAL ANALYSIS \\ IN A NUTRITIONAL INTERVENTION PROGRAM \\ Camila Neves Rodrigues ${ }^{a^{*}}$, Flávia Galvão Cândido ${ }^{b^{*}}$, \\ Rita de Cássia Gonçalves Alfenas ${ }^{*}$, Helen Hermana Miranda Hermsdorff ${ }^{*}$ \\ acamila.n.rodrigues@ufv.br, bflaviagcandido@hotmail.com, cralfenas@ufv.br, dhelenhermana@ufv.br \\ *Departamento de Nutrição e Saúde, Universidade Federal de Viçosa - Viçosa (MG), Brasil
}

Data de recebimento do artigo: 28/07/2014 Data de aceite do artigo: 21/01/2015

\section{RESUMO}

Introdução: A resistência à insulina (RI) é frequentemente associada ao diabetes tipo 2 (DM2), que se apresenta como uma morbidade de importante impacto devido as suas possíveis complicaçóes. Objetivos: Identificar indivíduos com RI e DM2 e avaliar as possíveis diferenças em suas características sociodemográficas, antropométricas e de composição corporal, clínicas e de comportamento alimentar. Materiais e métodos: Trata-se de um estudo transversal observacional. Participaram do estudo 68 pacientes atendidos em um programa de intervenção nutricional visando à prevenção e ao tratamento de doenças cardiovasculares. Os dados foram coletados de prontuários referentes à primeira consulta. Para identificação de indivíduos com RI utilizou-se o índice HOMA-IR>2,7. Resultados: Vinte e sete indivíduos foram classificados como grupo controle, 21 com RI e não diabéticos e 20 com DM2. Tanto os resistentes à insulina quanto os diabéticos tiveram maiores valores de IMC $(\mathrm{p}=00001)$, gordura corporal $(\mathrm{p}=0,001)$, perímetro da cintura $(\mathrm{p}=0,001)$ e do quadril $(\mathrm{p}=0,002)$ quando comparados ao grupo controle. Foi observada maior prevalência de hipertensão arterial $(70 \%)$ nos diabéticos. Os resistentes à insulina tiveram menores valores de HDL $(\mathrm{p}=0,009)$ e maiores de ácido úrico $(\mathrm{p}=0,033)$, em comparaçáo ao grupo controle, e maiores de LDL ( $\mathrm{p}=0,031)$, quando comparados aos diabéticos. Esses também relataram maior uso de banha animal $(15,8 \%)$ e mudanças de hábitos alimentares nos finais de semana $(84,2 \%)$. Conclusóes: Os resultados deste estudo ressaltam a importância da detecção precoce da RI e de programas de intervenção nutricional, visando ao controle de peso corporal e combate à hipertensão arterial tanto em diabéticos como em resistentes à insulina.

Palavras-chave: Diabetes mellitus; resistência à insulina; comportamento alimentar; doenças cardiovasculares.

\section{ABSTRACT}

Introduction: Insulin resistance (IR) is often associated with diabetes type 2 (DM2), that presents itself as a morbidity of important impact due to its possible complications. Objectives: To identify individuals IR and DM2 and to evaluate possible differences in their sociodemographic, anthropometrics, of body and clinical compositions, and feeding behavior characteristics. Materials and methods: This is an observational cross-sectional study. The study included 68 patients enrolled in a nutritional intervention program for the prevention and treatment of cardiovascular diseases. Data were collected from records concerning the first query. To identify insulin-resistant individuals it was used the HOMA-IR $>2,7$. Results: Twenty seven individuals were classified as the control group, 21 as insulin resistant and non-diabetics and 20 as diabetics. Both insulin resistant and diabetics had higher BMI $(p=0,001)$, body fat $(p=0,001)$, circumference of waist $(p=0,001)$ and hip $(p=0,002)$, compared to the control group. More hypertensive diabetics $(70 \%)$ were observed. The insulin resistant had lower levels of HDL $(\mathrm{p}=0,009)$ and higher of uric acid $(\mathrm{p}=0,033)$ compared to controls and higher LDL $(\mathrm{p}=0,031)$ when compared to diabetic. Those reported greater use of animal lard $(15,8 \%)$ and changing habits on weekends $(84,2 \%)$. Conclusions: The results 
of this study emphasize the importance of early detection of IR and nutrition intervention programs aiming at the weight control and combat hypertension in diabetic as in insulin resistant.

Keywords: Diabetes mellitus; insulin resistance; feeding behavior; cardiovascular diseases.

\section{Introdução}

O diabetes melito é definido como um distúrbio no metabolismo glicídico resultante de diversas causas, no qual o hormônio insulina tem sua secreção e/ou ação comprometida, levando a uma hiperglicemia característica da doença ${ }^{1}$.

O diabetes melito (DM) possui duas diferentes formas, que variam de acordo com a causa: o tipo 1 e o tipo 2. O diabetes melito tipo 1 (DM1) se caracteriza pela ausência da secreção da insulina causada pela destruição das células $\beta$ do pâncreas, enquanto que o diabetes melito tipo 2 (DM2) apresenta deficiência parcial da insulina ou mesmo comprometimento de sua ação ${ }^{2}$.

No Brasil (DATASUS) ${ }^{3}$, foram cadastrados 33.858.042 casos de DM no ano de 2012, o que correspondeu a aproximadamente $17,5 \%$ da população estimada para esse mesmo ano. Tais dados estáo de acordo com grande prevalência mundial da doença, que tem aumentado tanto em países em desenvolvimento como em países desenvolvidos 4 .

Entre as complicações do DM, encontram-se (além da descompensação glicêmica) as chamadas complicações crônicas, como retinopatias, neuropatias, cardiopatias, nefropatias e pé diabético ${ }^{1}$. Ademais, o DM2 se associa a diversas outras doenças crônicas não transmissíveis, como a obesidade, dislipidemias, hipertensão arterial, síndrome metabólica e doenças cardiovasculares ${ }^{5,6}$.

A resistência à insulina $(\mathrm{RI})$ precede o DM pela ineficiente ação da insulina plasmática, em concentraçoóes normais, o que resulta em uma hiperinsulinemia compensatória para manter as concentraçóes de glicemia dentro da normalidade, no entanto, quando o equilíbrio é rompido, instala-se o DM. Além de comprometer a adequada captaçáo periférica da glicose, a RI pode comprometer a gliconeogênese hepática e a inibição da produção de lipoproteína de muito baixa densidade $(\mathrm{VLDL})^{7,8}$.

Há variáveis que comumente são encontradas alteradas tanto em diabéticos como em resistentes à insulina, como o excesso de peso, perímetro da cintura, colesterol total, triglicerídeos; em diabéticos são relatadas também concentraçôes de LDL elevadas e HDL reduzido"10. Assim, muitos são os estudos que mostram a resistência insulínica como um importante fator de risco para diabetes, mas ainda são escassos aqueles que comparam características relevantes entre resistentes à insulina e diabéticos.

Atualmente, há métodos para se detectar indivíduos insulinorresistentes, como o teste de tolerância endovenosa à glicose e técnicas de clamp euglicêmico-hiperinsulinêmico, sendo que este é considerado padrâo-ouro e maior acurácia, porém é invasivo e caro ${ }^{6}$. O índice HOMA-IR (Homeostasis Model Assessment Insulin Resistance) constitui um modelo matemático que utiliza dados bioquímicos de insulinemia e glicemia de jejum para cálculo da resistência insulínica 6 . Assim, considerando principalmente as possíveis complicaçôes sérias decorrentes do diabetes, a detecção de indivíduos resistentes à insulina é essencial para controle metabólico do indivíduo ${ }^{11,12}$.

Por sua vez, a dieta habitual, o valor calórico e aporte de nutrientes de um plano alimentar habitual tem um papel importante na prevenção e tratamento das doenças crônicas não transmissíveis. A ingestão de uma dieta saudável, caracterizada por um consumo adequado de alimentos de todos os grupos alimentares, bem como o baixo consumo de gorduras saturadas, álcool e o excesso de açúcares exercem um efeito protetor contra tais doenças ${ }^{13}$.

Assim, este estudo teve como objetivo identificar indivíduos resistentes à insulina e DM2 entre usuários de um programa intervenção nutricional, e avaliar as possíveis diferenças em suas características sociodemográficas, antropométricas e de composiçáo corporal, clínicas e de seu comportamento alimentar.

\section{Metodologia}

\section{Sujeitos}

Trata-se de um estudo observacional transversal, no qual foram utilizados dados registrados na primeira consulta de indivíduos participantes do Programa de Atenção à Saúde Cardiovascular da Universidade Federal de Viçosa (Procardio-UFV), correspondente ao período de fevereiro de 2012 a fevereiro de 2014. O Procardio-UFV é um programa de intervenção nutricional, no qual os participantes recebem um tratamento e acompanhamento nutricional personalizado, incluindo atividades de educação nutricional, monitoramento dos dados antropométricos, bioquímicos e dos hábitos alimentares, além da prescrição 
dietética e encaminhamento a outros profissionais de saúde, quando necessário. $\mathrm{O}$ presente estudo está incluído no Registro Brasileiro de Ensaios Clínicos (ReBEC), no identificador primário: RBR-5n4y2g.

Os critérios de inclusão foram aqueles requeridos pelo próprio projeto Procardio-UFV, que são: ter idade igual ou superior a 20 anos e apresentar um ou mais dos critérios: sobrepeso ou obesidade (IMC $\geq 25,0 \mathrm{~kg}$ / $\mathrm{m}^{2}$ ), hipertrigliceridemia $(\geq 150 \mathrm{mg} / \mathrm{dL})$, hipercolesterolemia $(\geq 200 \mathrm{mg} / \mathrm{dL}$ ), HDL colesterol baixo (homem $\leq 40 \mathrm{mg} / \mathrm{dL}$ e mulher $\leq 50 \mathrm{mg} / \mathrm{dL}$ ), pressão arterial $\geq$ $130 / 85 \mathrm{mmHg}$ ou hipertensão diagnosticada, glicemia $\geq 100 \mathrm{mg} / \mathrm{dL}$, DM diagnosticada e/ou encaminhamento médico. Ademais, para o presente estudo, foram selecionados aqueles pacientes que possuíam dados iniciais glicemia e insulinemia de jejum, para sua categorização de acordo com ocorrência ou não de RI ou DM2.

Este estudo foi previamente aprovado pelo Comitê de Ética em Pesquisas com Seres Humanos da UFV (Of. $\left.\mathrm{N}^{\circ} 066 / 2012 / \mathrm{CEP}-U F V\right)$. Todos aqueles que concordaram em participar do estudo assinaram o Termo de Consentimento Livre e Esclarecido, de acordo com a resolução CNS 466/2012. Dos 239 voluntários do ProcardioUFV, foram selecionados 68 indivíduos os quais possuíam dados iniciais de insulinemia e glicemia de jejum. Estes foram categorizados em três grupos: controle (aqueles não resistentes à insulina e não diabéticos), grupo de resistentes à insulina e não diabéticos, e grupo de diabéticos.

\section{Diagnóstico de resistência à insulina e de diabetes tipo 2}

Foram considerados diabéticos aqueles indivíduos que relataram o DM como o motivo da consulta e faziam uso de medicamento relacionado, hipoglicemiante oral ou insulina, ou ainda de acordo com os exames bioquímicos, a glicemia de jejum e a hemoglobina glicada, dos quais se utilizou como ponto de corte para glicemia de jejum usou-se $\geq 126 \mathrm{mg} / \mathrm{dL}$ de acordo com a I Diretriz Brasileira de Diagnóstico e Tratamento da Síndrome Metabólica ${ }^{14}$ e para hemoglobina glicada $\geq 6,5 \%{ }^{15}$.

A classificação de RI foi determinada mediante o método de homeostase glicêmica (HOMA-IR), calculado pelo produto da insulina de jejum $(\mu \mathrm{UI} / \mathrm{mL})$ e da glicemia de jejum (mmol/L) dividido por $22,5^{16}$. Foram considerados resistentes à insulina os indivíduos que apresentavam valor de HOMA-IR maior de 2,7 ${ }^{17}$.

\section{Antropometria e composição corporal}

As medidas antropométricas coletadas foram peso, estatura, perímetro da cintura na cicatriz umbilical e do quadril, além do percentual de gordura. O peso corporal foi feito em balança digital eletrônica (Toledo 2098PP, São Bernardo do Campo, Brasil), com capacidade máxima de $200 \mathrm{~kg}$ e precisão de $50 \mathrm{~g}$. A estatura foi determinada em estadiômetro (Stanley, CMS, Inglaterra), com extensão máxima de $2 \mathrm{~m}$, precisão de $0,5 \mathrm{~mm}$. O Índice de Massa Corporal (IMC) foi calculado de acordo com a fórmula de peso $(\mathrm{kg})$ dividido pela altura $(\mathrm{m})$ ao quadrado. $\mathrm{O}$ mesmo foi utilizado para classificação do estado nutricional segundo a Organização Mundial de Saúde ${ }^{18}$ e Lipschitz ${ }^{19}$ para adultos e idosos (maiores de 60 anos), respectivamente.

O perímetro da cintura foi aferido na posição da cicatriz umbilical, e o perímetro do quadril feito mediante a maior porçáo glútea (como ponto anatômico de referência), ambos realizados com fita métrica inelástica ${ }^{18}$. O ponto de corte utilizado para a obesidade abdominal foi o perímetro da cintura $\geq 88 \mathrm{~cm}$ para mulheres e $\geq 102$ cm para homens ${ }^{20}$.

O percentual de gordura total foi determinado mediante impedância bioelétrica (Biodynamics 310 model, Washington, USA) de acordo um protocolo padronizado $^{21}$.

\section{Pressão arterial e marcadores metabólicos}

As pressôes arteriais sistólica e diastólica foram aferidas por meio do esfignomanômetro mecânico de coluna de mercúrio (BIC, São Paulo, Brasil), com aproximação de $2 \mathrm{mmHg}$, conforme técnica descrita na VI Diretrizes Brasileiras de Hipertensão ${ }^{22}$. Os pontos de cortes utilizados para classificação de hipertensão arterial foram $\geq 130 \mathrm{mmHg}$ ou $\geq 85 \mathrm{mmHg}$, segundo a I Diretriz Brasileira de Diagnóstico e Tratamento da Síndrome Metabólica $^{14}$.

Os dados de glicemia e insulinemia de jejum bem como, das concentrações séricas de triglicerídeos, colesterol total e fraçóes, ácido úrico, proteína $\mathrm{C}$ reativa ultrassensível (PCR-us), contagem de leucócitos totais foram coletados dos prontuários referentes ao início do acompanhamento no Procardio-UFV. Tais análises bioquímicas foram realizadas no Laboratório de Análises Clínicas da Divisão de Saúde da UFV, de acordo com protocolo padronizado pelo mesmo.

\section{Comportamento alimentar}

O comportamento alimentar foi avaliado por meio de perguntas específicas abertas e fechadas vindas do próprio questionário de atendimento, sendo abordadas questões como o local onde são feitas as grandes refeições (almoço e jantar), por quem são comprados os 
alimentos e preparadas as refeições, percepção da quantidade de sal utilizado e acréscimo deste no prato, tipo de gordura utilizada nas preparaçóes (óleo vegetal ou gordura animal). Além disso, foi calculado o consumo per capita mensal de açúcar dividindo-se o consumo mensal relatado pelo número de pessoas na casa, investigado o uso e tipo de adoçante, mudanças de hábitos alimentares em finais de semana e associaçóes entre o estado emocional e a ingestão alimentar.

\section{Outras variáveis}

Foram avaliadas variáveis sociodemográficas: sexo, idade, tipo de vínculo com a UFV e renda familiar. As variáveis relativas ao estilo de vida do paciente investigadas foram a prática de atividade física ( $\mathrm{sim} / \mathrm{não})$, o tabagismo ( $\operatorname{sim} /$ não) e o consumo de bebidas alcóolicas ( $\mathrm{sim} / \mathrm{naao}$ ). Ainda, os indivíduos relataram sobre o uso de hipoglicemiante oral e/ou insulina e outros medicamentos obtidos por meio de um questionário específico.

\section{Análise estatística}

As variáveis contínuas foram testadas quanto à sua normalidade mediante teste de Shapiro-Wilk. As variáveis foram descritas como média e desvio padrão $\pm \mathrm{DP}$ ou mediana, e intervalo interquartílico $(25 / 75 \%)$ ou ainda como valor absoluto (percentual).

As diferenças entre os grupos para as variáveis contínuas foram detectadas pela aplicação de ANOVA de um fator, seguida do teste de Tukey (variáveis paramétricas), ou Kruskal-Wallis, seguido por teste de Dunn (variáveis não paramétricas). As diferenças entre os grupos para as variáveis categóricas foram detectadas mediante a aplicação do teste Qui Quadrado. As análises foram conduzidas com o auxílio do programa Statistical Package for the Social Sciences (SPSS, versão 20.0). Um nível $\alpha$ menor que $5 \%$ foi considerado significativo.

\section{Resultados}

Participaram do estudo 68 indivíduos, sendo 26 homens e 42 mulheres. Destes, 27 foram classificados como não resistentes à insulina e não diabéticos (grupo controle), 21 apresentaram resistência, mas não tinham diabetes (grupo dos resistentes à insulina) e 20 apresentaram diabetes (grupo diabéticos) (Tabela 1 ).

Observou-se que os resistentes à insulina tiveram mediana da idade menor que a dos diabéticos. A maior parte da população estudada apresentou excesso de peso, segundo a classificação pelo IMC, sendo sua ocorrência significantemente maior entre os resistentes à insulina e os diabéticos. O grupo controle apresentou medidas da perímetro da cintura, do quadril e de gordura corporal significantemente menor em relaçáo aos dois outros grupos. Além disso, a pressão arterial sistólica foi mais elevada entre os diabéticos em relação ao grupo controle e resistentes à insulina.

As diferenças na distribuição da idade entre os grupos também foi observada quando se dividiu a amostra em categorias de idade, sendo o grupo dos diabéticos aquele que apresentou maior percentual de idosos (Figura 1, A). Também houve maior ocorrência de excesso de peso, segundo IMC, em resistentes à insulina e diabéticos (Figura 1, B) e uma menor ocorrência de percentual de gordura elevado entre os indivíduos do grupo controle, quando comparado aos resistentes à insulina e diabéticos (Figura 1, C). Por sua vez, uma maior ocorrência de elevação da pressão arterial entre os diabéticos e resistentes à insulina foi apresentada, quando comparados ao grupo controle (Figura 1, D).

Em relação aos marcadores metabólicos, observou-se que a insulinemia de jejum e o índice HOMA-IR foram maiores entre o grupo de resistentes à insulina e diabéticos comparado ao grupo controle, como esperado. A glicemia de jejum foi maior entre os diabéticos comparado tanto ao controle quanto aos resistentes à insulina (Tabela 2). Por sua vez, as concentraçóes de HDL foram significantemente maiores no grupo controle em relação aos resistentes à insulina, enquanto as concentraçóes de ácido úrico foram menores. Já o LDL foi maior entre os resistentes à insulina quando comparados aos diabéticos, enquanto as concentraçóes de colesterol total foram maiores entre os indivíduos do grupo controle quando comparados aos diabéticos.

Em relação ao uso de medicamentos, os indivíduos diabéticos consumiram mais medicamentos em relação aos demais. Houve um maior consumo de anti-hipertensivos orais e sublinguais pelos diabéticos em relação aos resistentes à insulina e ao grupo controle. Observouse também que o uso de redutores de colesterol foi menor entre os resistentes à insulina (Tabela 3).

Quando as características do comportamento alimentar da amostra foram avaliadas, observou-se a maior utilização de adoçante não nutritivo por diabéticos, sendo que todos relataram fazer uso de algum edulcorante. $\mathrm{O}$ tipo de adoçante mais utilizado foi aqueles à base de sacarina e ciclamato monossódico e o menos utilizado, a sucralose. Contudo, não houve diferença no consumo entre os grupos do estudo para os diferentes tipos de adoçante.

Além disso, foi observado que tanto o uso de gordura quanto o de óleo vegetal é maior entre os resistentes à insulina e que o uso de somente óleo vegetal é maior no grupo controle quando comparado aos demais. O grupo de 
resistentes à insulina mudaram mais seus hábitos alimentares aos finais de semana em relação aos demais (Tabela 4).

\section{Discussão}

O presente estudo avaliou as diferenças existentes entre indivíduos sem e com a ocorrência de RI ou DM2, atendidos em um programa de intervenção nutricional que visa a prevenção e/ou tratamento de doenças cardiovasculares.
Nesse contexto, nossos resultados evidenciaram aumento da idade no grupo de indivíduos diabéticos em relação aos resistentes à insulina. A RI é um distúrbio que precede o $\mathrm{DM} 2^{23}$ e tal fato justifica os menores valores de idade dos indivíduos resistentes à insulina quando comparado aos diabéticos, como foi observado. Além disso, é comum o DM2 não ser detectado precocemente e que indivíduos diabéticos só sejam encaminhados para programas de intervenção após anos de apresentação assintomática da doença ${ }^{24}$.

Tabela 1: Perfil antropométrico e de composição corporal, sociodemográfico da amostra de acordo com os grupos do estudo, Procardio-UFV, 2012-2014.

\begin{tabular}{|c|c|c|c|c|}
\hline \multirow{2}{*}{ Variáveis } & Controle & Resistentes à insulina & Diabéticos & \multirow{2}{*}{ Valor de $\mathrm{P}$} \\
\hline & $(\mathrm{n}=27)$ & $(n=21)$ & $(\mathrm{n}=20)$ & \\
\hline Idade (anos) & $49(25-55) \mathrm{ab}$ & $36(21,5-55) a$ & $57(54,3-63,8) b$ & 0,005 \\
\hline \multicolumn{5}{|l|}{ Sexo } \\
\hline Homem & $7(26,9 \%)$ & $9(45 \%)$ & $10(47,6 \%)$ & \multirow[t]{2}{*}{0,117} \\
\hline Mulher & $20(74,1 \%)$ & $11(55 \%)$ & $11(52,4 \%)$ & \\
\hline \multicolumn{5}{|l|}{ Vínculo } \\
\hline Servidor & $8(29,6 \%)$ & $6(28,6 \%)$ & $9(45 \%)$ & \multirow{4}{*}{0,843} \\
\hline Estudante & $11(40,8 \%)$ & $8(38,1 \%)$ & $2(10 \%)$ & \\
\hline Familiar & $8(29,6 \%)$ & $7(33,3 \%)$ & $8(40 \%)$ & \\
\hline Cooperação & $0(0 \%)$ & $0(0 \%)$ & $1(5 \%)$ & \\
\hline \multicolumn{5}{|l|}{ Renda familiar } \\
\hline Não informada & $2(10 \%)$ & $0(0 \%)$ & $0(0 \%)$ & \multirow{3}{*}{0,483} \\
\hline Até 4 salários & $14(70 \%)$ & $7(53,8 \%)$ & $13(81,2 \%)$ & \\
\hline Maior que 4 salários & $4(20 \%)$ & $6(46,2 \%)$ & $3(18,8 \%)$ & \\
\hline $\operatorname{IMC}\left(\mathbf{k g} / \mathbf{m}^{2}\right)$ & $26,8 \pm 3,96 a$ & $31,9 \pm 4,5 b$ & $31,1 \pm 6,4 \mathrm{~b}$ & 0,001 \\
\hline Perímetro da cintura $(\mathrm{cm})$ & $91,7 \pm 11,4 \mathrm{a}$ & $103,9 \pm 12,9 b$ & $104,7 \pm 13,6 b$ & 0,001 \\
\hline Perímetro do quadril (cm) & $99,5(95,3-104,8) \mathrm{a}$ & $112(99,5-119,5) b$ & $105(97-110) b$ & 0,005 \\
\hline Massa magra (kg) & $48,2 \pm 9,6$ & $55,3 \pm 14,8$ & $53,7 \pm 9,3$ & 0,178 \\
\hline Gordura corporal $(\mathbf{k g})$ & $20,1(16,1-24,5) a$ & $30,3(23,5-35,3) b$ & $27,6(23,4-32,4) b$ & $<0,001$ \\
\hline Pressão arterial sistólica (mmHg) & $120(112,5-120) \mathrm{a}$ & $120(110-130) a$ & $130(120-140) b$ & 0,001 \\
\hline Pressão arterial diastólica (mmHg) & $80(70-80)$ & $80(70-90)$ & $80(80-90)$ & 0,503 \\
\hline \multicolumn{5}{|l|}{ Tabagismo } \\
\hline Nunca fumou & $22(81,5 \%)$ & $17(81 \%)$ & $11(55 \%)$ & \multirow{3}{*}{0,815} \\
\hline Já fumou & $4(14,8 \%)$ & $3(14,3 \%)$ & $7(35 \%)$ & \\
\hline Fumante & $1(3,7 \%)$ & $1(4,7 \%)$ & $2(10 \%)$ & \\
\hline \multicolumn{5}{|l|}{ Atividade física } \\
\hline Sim & $19(70,4 \%)$ & $11(55 \%)$ & $12(57,1 \%)$ & \multirow[t]{2}{*}{0,329} \\
\hline Nāo & $8(29,6 \%)$ & $9(45 \%)$ & $9(42,9 \%)$ & \\
\hline Horas de sono (hs) & $3(3-3)$ & $3(3-4,25)$ & $2(2,25-4,0)$ & 0,102 \\
\hline \multicolumn{5}{|l|}{ Consumo habitual de álcool } \\
\hline Não bebe & $11(42,3 \%)$ & $9(45 \%)$ & $8(42,1 \%)$ & \multirow{3}{*}{0,936} \\
\hline Eventualmente & $14(53,8 \%)$ & $10(50 \%)$ & $10(52,6 \%)$ & \\
\hline Diariamente & $1(3,8 \%)$ & $11(5 \%)$ & $1(5,3 \%)$ & \\
\hline
\end{tabular}

Valores expressos em média \pm DP, mediana (25/75\%) ou em números absolutos (percentual). p: resultados das comparaçōes entre os grupos experimentais, de acordo com os testes ANOVA de um fator ou Kruskal-Wallis (variáveis numéricas paramétricas e não paramétricas, respectivamente) ou pelo teste de Qui Quadrado (variáveis categóricas). Resultados em negrito expressam diferença significativa, sendo que letras distintas representam diferença entre os grupos $(\mathrm{p}<0,05)$, de acordo com os testes de Tukey e Dunn (variáveis paramétricas e não paramétricas, respectivamente). 

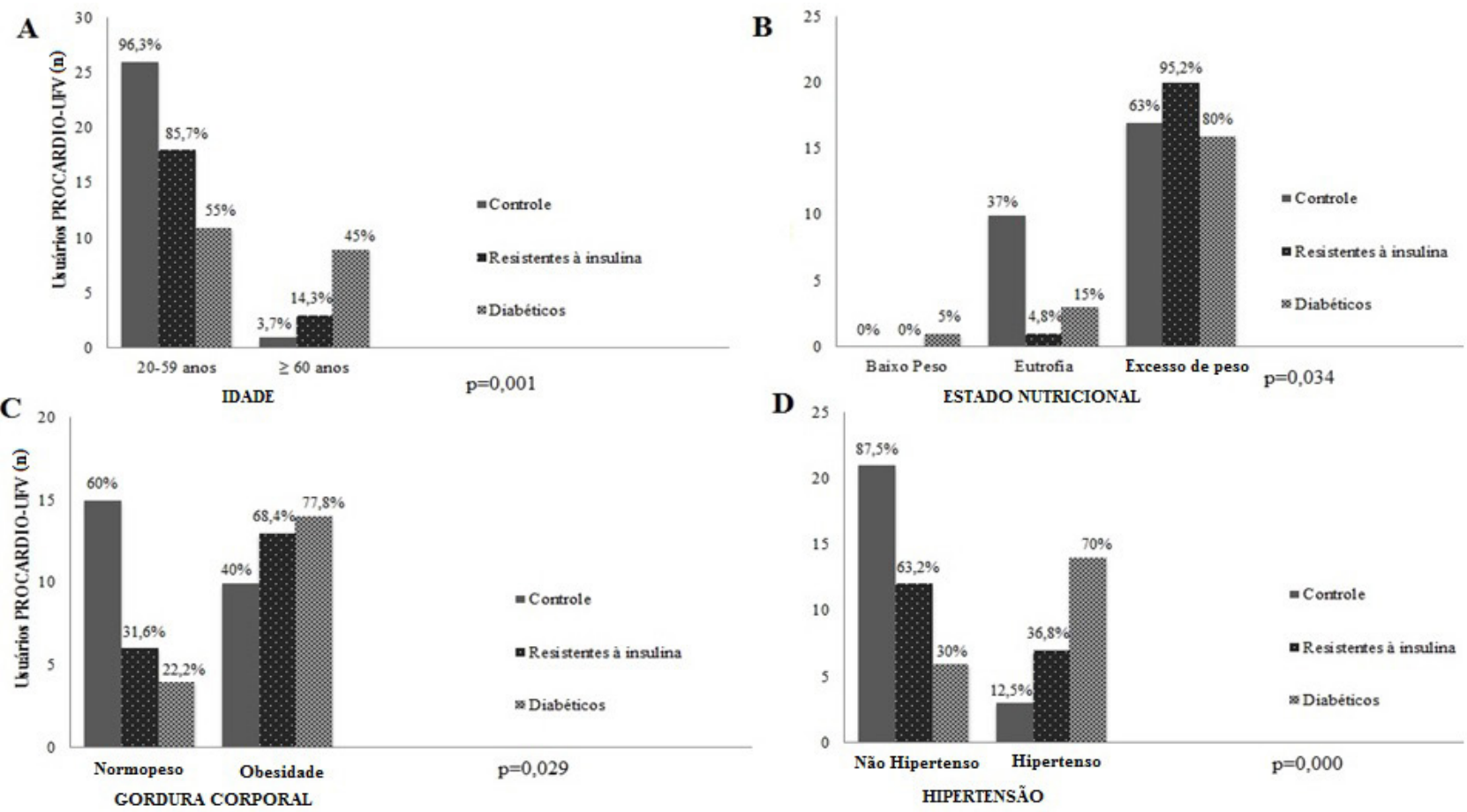

Figura 1: Distribuição das características relevantes entre os grupos de estudo na amostra avaliada, Procardio-UFV, 2012-2014. Classificação dos indivíduos dos grupos controle, resistentes à insulina e diabéticos em relação à idade (A), estado nutricional (B), percentual de gordura corporal (C) e presença de hipertensão arterial (D) na amostra avaliada. p: valores dos resultados das comparações pelo teste de Qui Quadrado.

Tabela 2: Perfil de marcadores metabólicos da amostra de acordo com os grupos do estudo, Procardio-UFV, $2012-2014$.

\begin{tabular}{|c|c|c|c|c|}
\hline \multirow{2}{*}{ Variáveis } & Controle & Resistentes à insulina & Diabéticos & \multirow{2}{*}{$\mathbf{p}$} \\
\hline & $(\mathrm{n}=27)$ & $(n=21)$ & $(n=20)$ & \\
\hline Insulinemia de jejum $(\mathrm{mU} / \mathrm{mL})$ & $7,9(5,6-9,8)^{a}$ & $15,1(13,2-20,8)^{b}$ & $11,3(9,2-18,2)^{\mathrm{b}}$ & $<0,001$ \\
\hline Glicemia jejum (mg/dL) & $84(79,5-87)^{\mathrm{a}}$ & $94(85-100)^{\mathrm{a}}$ & $121(92,75-139,5)^{\mathrm{b}}$ & $<0,001$ \\
\hline Índice HOMA-IR & $1,5(1,2-2,1)^{\mathrm{a}}$ & $3,5(3,1-5,3)^{b}$ & $3,9(2,4-5,0)^{b}$ & $<0,001$ \\
\hline Triglicerídeos (mg/dL) & $116,5(91,5-197,8)$ & $129(107,5-157,5)$ & $144(107,5-157,5)$ & 0,599 \\
\hline Colesterol total (mg/dL) & $214 \pm 31,5^{\mathrm{b}}$ & $197 \pm 30,6^{\mathrm{ab}}$ & $187,6 \pm 44,5^{\mathrm{a}}$ & 0,041 \\
\hline $\mathrm{LDL}(\mathrm{mg} / \mathrm{dL})$ & $122,8 \pm 31,9^{\mathrm{ab}}$ & $125,5 \pm 29,1^{\mathrm{b}}$ & $100,5 \pm 32,4^{\mathrm{a}}$ & 0,031 \\
\hline HDL (mg/dL) & $51(45-67,5)^{b}$ & $39(31,5-54)^{\mathrm{a}}$ & $44,6(38,5-54,8)^{\mathrm{ab}}$ & 0,015 \\
\hline VLDL (mg/dL) & $24,2(18,6-44,8)$ & $23,8(16,6-31)$ & $25,4(21,9-31,5)$ & 0,722 \\
\hline PCR-us (mg/L) & $1,9(1,3-8,3)$ & $2,5(0,7-5,9)$ & $1,6(0,5-4,6)$ & 0,446 \\
\hline Leucócitos $\left(/ \mathrm{mm}^{3}\right)$ & $5.792,3 \pm 1.460,1$ & $6.385,5 \pm 1.748,4$ & $6.900 \pm 2.016,5$ & 0,104 \\
\hline Ácido úrico & $3,2(2,6-4,6)^{\mathrm{a}}$ & $4,6(3,4-6,8)^{\mathrm{b}}$ & $3,7(2,9-6,2)^{\mathrm{ab}}$ & 0,033 \\
\hline
\end{tabular}

HOMA-IR: Homeostatic Model Assessment of Insulin Resistence. Valores expressos em média \pm DP, mediana (25/75\%). p: resultados das comparaçốes entre os grupos experimentais, de acordo com os testes ANOVA de um fator ou Kruskal-Wallis (variáveis numéricas paramétricas e não paramétricas, respectivamente). Resultados em negrito expressam diferença significativa, sendo que letras distintas representam diferença entre os grupos ( $\mathrm{p}<0,05)$, de acordo com os testes de Tukey e Dunn (variáveis paramétricas e năo paramétricas, respectivamente). 
Tabela 3: Uso de medicamentos da amostra de acordo com os grupos do estudo, Procardio-UFV, 2012-2014.

\begin{tabular}{|c|c|c|c|c|}
\hline \multirow{2}{*}{ Variáveis } & Controle & Resistentes à insulina & Diabéticos & \multirow{2}{*}{$\mathbf{p}$} \\
\hline & $(n=27)$ & $(n=21)$ & $(n=20)$ & \\
\hline \multicolumn{5}{|c|}{ Uso de medicamentos } \\
\hline Não & $8(29,6 \%)$ & $6(28,6 \%)$ & $0(0 \%)$ & \multirow[t]{2}{*}{0,025} \\
\hline Sim & $19(70,4 \%)$ & $15(71,4 \%)$ & $20(100 \%)$ & \\
\hline \multicolumn{5}{|l|}{ Aspirina } \\
\hline Não & $26(96,3 \%)$ & $21(100 \%)$ & $19(95 \%)$ & \multirow[t]{2}{*}{0,610} \\
\hline Sim & $1(3,7 \%)$ & $0(0 \%)$ & $1(5 \%)$ & \\
\hline \multicolumn{5}{|c|}{ Outros analgésicos } \\
\hline Não & $26(96,3 \%)$ & $21(100 \%)$ & $20(100 \%)$ & \multirow[t]{2}{*}{0,463} \\
\hline Sim & $1(3,7 \%)$ & $0(0 \%)$ & $0(0 \%)$ & \\
\hline \multicolumn{5}{|c|}{ Anti-hipertensivo oral } \\
\hline Não & $19(70,4 \%)$ & $14(66,7 \%)$ & $6(30 \%)$ & \multirow[t]{2}{*}{0,013} \\
\hline Sim & $8(29,6 \%)$ & $7(33,3 \%)$ & $14(70 \%)$ & \\
\hline \multicolumn{5}{|c|}{ Anti-hipertensivo sublingual } \\
\hline Não & $27(100 \%)$ & $20(95,2 \%)$ & $16(80 \%)$ & \multirow[t]{2}{*}{0,034} \\
\hline Sim & $0(0 \%)$ & $1(4,8 \%)$ & $4(20 \%)$ & \\
\hline \multicolumn{5}{|c|}{ Redutores de colesterol } \\
\hline Não & $15(55,6 \%)$ & $17(81 \%)$ & $8(40 \%)$ & \multirow[t]{2}{*}{0,026} \\
\hline Sim & $12(44,4 \%)$ & $4(19 \%)$ & $12(60 \%)$ & \\
\hline \multicolumn{5}{|l|}{ Fibratos } \\
\hline Não & $26(96,3 \%)$ & $19(95 \%)$ & $21(100 \%)$ & \multirow[t]{2}{*}{0,610} \\
\hline Sim & $1(3,7 \%)$ & $1(5 \%)$ & $0(0 \%)$ & \\
\hline \multicolumn{5}{|c|}{ Tranquilizantes ou indutores } \\
\hline de sono & & & & \multirow{3}{*}{0,459} \\
\hline Não & $23(92 \%)$ & $17(89,5 \%)$ & $16(80 \%)$ & \\
\hline Sim & $2(8 \%)$ & $2(10,5 \%)$ & $4(20 \%)$ & \\
\hline \multicolumn{5}{|c|}{ Antidepressivos } \\
\hline Não & $27(100 \%)$ & $18(90 \%)$ & $20(95,2 \%)$ & \multirow[t]{2}{*}{0,255} \\
\hline Sim & $0(0 \%)$ & $2(10 \%)$ & $1(4,8 \%)$ & \\
\hline \multicolumn{5}{|c|}{ Omeprazol } \\
\hline Não & $24(88,9 \%)$ & $19(90,5 \%)$ & $15(75 \%)$ & \multirow[t]{2}{*}{0,299} \\
\hline Sim & $3(11,1 \%)$ & $2(9,5 \%)$ & $5(25 \%)$ & \\
\hline \multicolumn{5}{|c|}{ Hormônio Tireóide } \\
\hline Não & $24(88,9 \%)$ & $21(100 \%)$ & $16(80 \%)$ & \multirow[t]{2}{*}{0,107} \\
\hline Sim & $3(11,1 \%)$ & $0(0 \%)$ & $4(20 \%)$ & \\
\hline \multicolumn{5}{|c|}{ Outros medicamentos } \\
\hline Não & $17(63 \%)$ & $15(71,4 \%)$ & $11(55 \%)$ & \multirow[t]{2}{*}{0,551} \\
\hline Sim & $10(37 \%)$ & $6(28,6 \%)$ & $9(45 \%)$ & \\
\hline
\end{tabular}

Valores expressos em números absolutos (percentual). p é derivado de resultados das comparaçôes entre os grupos experimentais pelo teste de qui-quadrado (variáveis categóricas). Resultados em negrito expressam diferença significativa ( $\mathrm{p}<0,05)$. 
Rodrigues C. N., Cândido F. G., Alfenas R. C. G., Hermsdorff H. H. M.

Tabela 4: Características alimentares da amostra em relação aos grupos do estudo, Procardio-UFV, 2012-2014.

\begin{tabular}{|c|c|c|c|c|}
\hline Variáveis & $\begin{array}{c}\text { Controle } \\
(\mathrm{n}=27)\end{array}$ & $\begin{array}{l}\text { Resistentes à insulina } \\
\qquad(\mathrm{n}=21)\end{array}$ & $\begin{array}{l}\text { Diabético } \\
(\mathrm{n}=20)\end{array}$ & $\mathbf{p}$ \\
\hline \multicolumn{5}{|l|}{ Faz refeiçóes em casa } \\
\hline Não & $5(19,2 \%)$ & $5(23,8 \%)$ & $3(15,8 \%)$ & \multirow{3}{*}{0,955} \\
\hline Sim & $16(61,5 \%)$ & $11(52,4 \%)$ & $16(84,2 \%)$ & \\
\hline Ambos & $5(19,2 \%)$ & $5(23,8 \%)$ & $0(0 \%)$ & \\
\hline \multicolumn{5}{|l|}{ Quem compra os alimentos } \\
\hline A própria pessoa & $21(80,8 \%)$ & $15(75 \%)$ & $13(65 \%)$ & \multirow[t]{2}{*}{0,233} \\
\hline Outro & $5(19,2 \%)$ & $5(25 \%)$ & $7(35 \%)$ & \\
\hline \multicolumn{5}{|l|}{ Quem prepara as refeiçóes } \\
\hline A própria pessoa & $17(65,4 \%)$ & $12(60 \%)$ & $9(47,4 \%)$ & \multirow[t]{2}{*}{0,237} \\
\hline Outro & $9(34,6 \%)$ & $8(40 \%)$ & $10(52,6 \%)$ & \\
\hline \multicolumn{5}{|l|}{ Uso de sal } \\
\hline Normal & $9(33,3 \%)$ & $9(47,4 \%)$ & $6(30 \%)$ & \multirow{3}{*}{0,357} \\
\hline Pouco & $11(40,7 \%)$ & $6(31,6 \%)$ & $13(65 \%)$ & \\
\hline Muito & $7(25,9 \%)$ & $4(21,1 \%)$ & $1(5 \%)$ & \\
\hline \multicolumn{5}{|l|}{ Acrescenta sal no prato } \\
\hline Não & $19(73,1 \%)$ & $11(55 \%)$ & $13(65 \%)$ & \multirow[t]{2}{*}{0,206} \\
\hline Sim & $7(26,9 \%)$ & $9(45 \%)$ & $7(35 \%)$ & \\
\hline \multicolumn{5}{|l|}{ Gordura usada nas preparaçóes } \\
\hline Óleo vegetal & $27(100 \%)$ & $16(84,2 \%)$ & $19(95 \%)$ & \multirow[t]{2}{*}{0,031} \\
\hline Banha+Óleo vegetal & $0(0 \%)$ & $3(15,8 \%)$ & $1(5 \%)$ & \\
\hline Consumo per capita mensal de açúcar & $1,35 \pm 0,28$ & $0,99 \pm 0,17$ & $2,15 \pm 0,41$ & 0,318 \\
\hline \multicolumn{5}{|l|}{ Uso de adoçante não nutritivos } \\
\hline Não & $15(55,6 \%)$ & $8(42,1 \%)$ & $0(0 \%)$ & \multirow[t]{2}{*}{0,000} \\
\hline Sim & $12(44,4 \%)$ & $11(57,9 \%)$ & $20(100 \%)$ & \\
\hline \multicolumn{5}{|l|}{ Tipo de adoçante náo nutritivo utilizado } \\
\hline Sacarina sódica e ciclamato de sódio & $7(58,3 \%)$ & $8(72,7 \%)$ & $11(55 \%)$ & \multirow{5}{*}{0,873} \\
\hline Esteviosídeo & $2(16,7 \%)$ & $1(9,1 \%)$ & $5(25 \%)$ & \\
\hline Sucralose & $0(0 \%)$ & $0(0 \%)$ & $1(5 \%)$ & \\
\hline Arpartame & $1(8,3 \%)$ & $0(0 \%)$ & $1(5 \%)$ & \\
\hline Não informado & $2(16,7 \%)$ & $2(18,2 \%)$ & $2(10 \%)$ & \\
\hline \multicolumn{5}{|l|}{$\begin{array}{l}\text { Associaçáo entre consumo alimentar e } \\
\text { estado emocional }\end{array}$} \\
\hline Não & $10(38,5 \%)$ & $8(42,1 \%)$ & $7(33,3 \%)$ & \multirow{2}{*}{0,936} \\
\hline Sim & $16(61,5 \%)$ & $11(57,9 \%)$ & $14(66,7 \%)$ & \\
\hline \multicolumn{5}{|l|}{$\begin{array}{l}\text { Mudanças de hábitos nos fins de } \\
\text { semanas }\end{array}$} \\
\hline Não & $13(48,1 \%)$ & $3(15,8 \%)$ & $9(47,4 \%)$ & \multirow[t]{2}{*}{0,035} \\
\hline Sim & $14(51,9 \%)$ & $16(84,2 \%)$ & $10(52,6 \%)$ & \\
\hline
\end{tabular}

Valores expressos em média \pm DP, mediana (25/75\%) ou em números absolutos (percentual). p: resultados das comparaçôes entre os grupos experimentais por ANOVA de um fator ou Kruskall (variáveis numéricas) ou qui-quadrado (variáveis categóricas). Resultados em negrito expressam diferença significativa $(\mathrm{p}<0,05)$. 
No presente estudo, indivíduos resistentes à insulina e diabéticos tiveram maiores valores de IMC, de perímetro do quadril, da cintura e peso de gordura corporal em relação aos controle. A RI é descrita como a principal alteraçáo metabólica da obesidade e está relacionada majoritariamente com o acúmulo de gordura abdominal ${ }^{25}$.Indivíduos com excesso de peso corporal e que apresentam maior proporção de tecido adiposo do tipo visceral produzem hormônios e citocinas, como resistina, IL-6 e fator de necrose tumoral alfa, que aumentam a RI por diminuir a capacidade de captação celular da glicose $\mathrm{e}^{25,26}$. Penaforte et al. ${ }^{12}$ evidenciaram valores significantemente maiores de perímetro da cintura em indivíduos resistentes à insulina quando comparados aos não resistentes, mas tal diferença não foi observada para o perímetro do quadril. Em outro estudo com indivíduos diabéticos, observou-se elevação nos valores de perímetro da cintura em $86,2 \%$ da amostra ${ }^{27}$. Esses resultados ressaltam a importância do perímetro da cintura como indicador do risco de $\mathrm{RI}^{21}$, sendo este correlacionado positivamente com IMC ${ }^{27}$.

Outro resultado interessante foi o maior número de hipertensos entre os diabéticos da nossa amostra em relação ao grupo controle, assim como maior uso de antihipertensivos e antihipertensivo sublingual entre os diabéticos. Faria et al..$^{28}$ e Martins e Araújo ${ }^{29}$ afirmaram que a hipertensão arterial é comumente relacionada a indivíduos obesos diabéticos tipo 2 , sendo que sua prevalência no diabético varia de $20 \%$ a $60 \%$, dependendo da classe, idade, etnia e presença de obesidade. Assim como para a aterosclerose, a hipertensão arterial é citada como fator influenciado pela $\mathrm{RI}^{25}$. Acredita-se que níveis elevados de insulina ativam o sistema nervoso simpático e promovem a retenção de sódio por alteraçôes no funcionamento da medula renal, ativação do sistema renina angiotensina aldosterona e aumento da reabsorção tubular de sódio, causando a hipertensão arterial em indivíduos obesos ${ }^{28,30}$. Apesar dos possíveis mecanismos, o papel de hiperinsulinemia na patogênese da hipertensão arterial na síndrome metabólica ainda não foi confirmado pelos estudos ${ }^{30}$. Outro mecanismo defendido por Reaven ${ }^{31}$ diz respeito ao aumento da concentração do inibidor endógeno da síntese do óxido nítrico em indivíduos hipertensos e resistentes à insulina, sendo que o óxido nítrico tem um papel vasodilatador, já que promove o relaxamento das paredes dos vasos. Acredita-se que outros fatores de origem genética estejam envolvidos na patogênese da hipertensão arterial associada à RI, contudo, ainda se sabe pouco a respeito ${ }^{30}$. Santos-Filha et al. ${ }^{32}$ observou alta prevalência $(76,9 \%)$ de hipertensão arterial em diabéticos sem, contudo, observar alterações nas médias dos componentes do perfil lipídico desses indivíduos; no entanto, esse último resultado foi relacionado ao fato do tratamento medicamentoso à base de estatinas, usado para tratamento das alteraçóes lipídicas, adotado pela maioria dos indivíduos da amostra.

Por sua vez, o DM2 e os resistentes à insulina podem apresentar concomitantemente, em maior proporçáo, um perfil lipídico aterogênico, com elevação de triglicerídeos, colesterol total, LDL, além de baixas concentrações de $\mathrm{HDL}^{12,31}$. No entanto, maiores concentraçóes de colesterol total foram encontradas no grupo controle em relação aos diabéticos. Uma explicação para tal resultado é o maior consumo de medicamentos redutores de colesterol (estatinas) pelos diabéticos, o que justifica também as menores concentrações de LDL encontrados nos indivíduos diabéticos quando comparado aos resistentes à insulina. Já as baixas concentraçóes de HDL encontradas no grupo de resistentes à insulina quando comparado ao grupo controle, poderiam estar associadas ao maior percentual de eutróficos entre o grupo controle, o que representa um fator de importante impacto para adequação da concentração do colesterol e suas fraçóes ${ }^{33}$. Ao mesmo tempo, Vasques et al. ${ }^{34}$ encontraram uma relação negativa entre HDL e o índice HOMA-IR, sugerindo a correlação entre os dois biomarcadores metabólicos.

Além desses fatores consideráveis nas diferenças encontradas no perfil lipídico dos grupos, alguns autores ressaltam a influência da RI na fisiopatologia da aterosclerose, já que há evidências de que a insulina inibe a produção de VLDL 1 (uma subclasse de VLDL); assim no diabetes, como na resistência à insulina, não ocorre essa inibiçáo, favorecendo a formaçáo do LDLcolesterol. Como o uso de medicamentos redutores de colesterol pelos diabéticos é maior, não foram encontradas médias elevadas de LDL, mas tal característica foi encontrada nos resistentes à insulina ${ }^{25,28,35}$. Vale ressaltar que, apesar de todas as discussóes supracitadas, as alteraçóes negativas no perfil lipídico observadas nos pacientes com RI podem ser devido às características do grupo controle do presente estudo. Todos os indivíduos da amostra participavam de um programa de intervenção nutricional para controle de doenças cardiovasculares e as chances de o grupo controle apresentar maior incidência de dislipidemias do que os demais é grande, devido aos critérios de inclusão do programa.

As maiores concentraçôes de ácido úrico encontradas no grupo dos resistentes à insulina, em relação ao controle, podem estar relacionadas ao fato de que a hiperuricemia é comumente associada à síndrome da $\mathrm{RI}^{36}$. $\mathrm{Abreu}^{37}$ evidenciou um número significantemente maior de RI entre os indivíduos hiperuricêmicos, sendo que a prevalência RI nestes indíviduos foi de $62,8 \%$. E necessário considerar também que a concentração de ácido úrico parece se manter elevada antes do diagnóstico do DM e reduzir com o tempo da doença ${ }^{38}$, o que pode explicar o fato de ter sido observado, neste estudo, 
o aumento da uricemia apenas nos resistentes à insulina, mas não nos diabéticos.

Mudanças de hábitos alimentares nos finais de semana e uso de banha animal nas preparaçóes foram mais relatados pelos resistentes à insulina. Penaforte et al. ${ }^{12}$, ao compararem mulheres obesas com e sem resistência à insulina, não encontraram diferença com relação a fome, saciedade e consumo alimentar ad libitum. No entanto, os resultados do presente estudo podem refletir sinais de uma má alimentação, assim como uma menor aderência a práticas de alimentação saudável, bem como possíveis reflexos de atitudes muitas vezes tomadas como aceitáveis. Oliveira e Franco ${ }^{39}$ encontraram que $94,2 \%$ de indivíduos diabéticos reconheciam a importância da alimentação no tratamento, e 48,3\% destes declaram sempre seguir as recomendações dietoterápicas, assim conclui-se que o papel da alimentação no tratamento do DM já vem sendo bem reconhecida e consolidada pelos indivíduos, diferentemente da RI. Assim, o conhecimento da população quanto à RI e a influência na alimentação podem contribuir para tomada de hábitos saudáveis. Além disso sabe-se que o uso de banha animal representa um maior consumo de gordura saturada e colesterol, que em excesso levam a alteraçóes no perfil lipídico, como encontrado justamente no grupo que relatou maior uso da banha: os resistentes à insulina.

Atualmente, as diretrizes para diabéticos ${ }^{40}$ não restringem o uso de sacarose, já que essa não aumenta a glicemia mais do que quantidades isocalóricas de amido. O que é recomendado é que o consumo seja feito em substituição a outras fontes de carboidratos. Apesar disso, grande parte dos pacientes diabéticos ainda utiliza adoçante nâo nutritivo no lugar da sacarose $\mathrm{e}^{40}$, como foi observado no presente estudo. Oliveira e Franco ${ }^{39}$ observaram grande consumo de adoçante não nutritivo em diabéticos, onde $76,7 \%$ da amostra relataram o uso desses adoçantes após o diagnóstico da doença e atribuíram a ela o motivo do uso. Saito et al. ${ }^{41}$ também evidenciaram o maior consumo de adoçante à base de ciclamato de sódio e sacarina sódica, o que é um fator relevante ao considerarmos que o aumento do consumo de sódio pode aumentar a pressão arterial e a grande proporção de hipertensos na amostra. Duarte e Oliveira $^{42}$ justificaram que o menor consumo de adoçantes dos tipos esteviosídeo e sucralose, apesar de suas características vantajosas como menor sabor amargo e bom poder adoçante, é decorrente do seu preço mais elevado. No entanto, neste estudo foi observado um consumo considerável do esteviosídeo pelos diabéticos da amostra; já a sucralose teve baixo consumo entre os grupos, principalmente o grupo controle e resistentes à insulina.

\section{Conclusão}

Em conclusão, os resultados do presente estudo indicam uma associação positiva entre indicadores de adiposidade e a resistência à insulina, e o diabetes tipo 2, com maior ocorrência de sobrepeso/obesidade. Além disso, a presença de hipertensão arterial foi maior nos diabéticos, ressaltando a necessidade de estratégias de intervenção nutricional de combate ao excesso de peso corporal e à hipertensão arterial nesse grupo. Finalmente, foi encontrado um pior perfil lipídico e uricemia em resistentes à insulina, caracterizado por maiores concentraçóes de LDL e ácido úrico, e menores de HDL. Desse modo, a detecção precoce da RI e o tratamento personalizado aos indivíduos desse grupo podem prevenir ou retardar o aparecimento do DM2 e das doenças cardiovasculares.

\section{Agradecimentos}

Aos voluntários participantes do estudo, à equipe do Setor de Nutrição da Divisão de Saúde-UFV pela assistência técnica, e às estagiárias voluntárias do ProcardioUFV pelo apoio na coleta de dados.

\section{Referências}

1. Brasil. Ministério da Saúde. Secretaria de Atenção à Saúde. Departamento de Atenção Básica. Estratégias para o cuidado da pessoa com doença crônica: diabetes mellitus. Brasília: Ministério da Saúde, 2013.

2. Soares MCC. Estado Nutricional e Padrão Alimentar de Idosos Diabéticos, [Dissertação de mestrado em Ciência da Saúde]. Coimbra: Faculdade de Medicina da Universidade de Coimbra, Universidade de Aveiro; 2009.61 p.

3. Brasil. Ministério da Saúde. Departamento de Informática do Sistema Único de Saúde (DATASUS). Brasília [s.d.] [acesso em: dez 2013]. Disponível em: http://www.datasus. gov.br.

4. Whiting DR, Guariguata L, Weil C, Shaw J. IDF diabetes atlas: global estimates of the prevalence of diabetes for 2011 and 2030. Diabetes Res Clin Pract 2011;94:311-21.

5. Brasil. Ministério da Saúde. Secretaria de Atenção à Saúde. Departamento de Atenção Básica. Diabetes Mellitus. Brasília: Ministério da Saúde, 2006.

6. Vasques ACJ, Rosado LEFPL, Alfenas RCG, Geloneze B. Análise crítica do uso dos índices do Homeostasis Model Assessment (HOMA) na avaliação da resistência à insulina e capacidade funcional das células beta-pancreáticas. Arq Bras Endocrinol Metab. 2008;52(1):32-39.

7. Rodrigues TC, Canani LH, Gross JL. Síndrome metabólica, resistência a ação da insulina e doença cardiovascular no 
diabete melito tipo 1. Arq Bras Cardiol 2010;94(1):134-39.

8. Madeira IR, Carvalho CNM, Gazolla FM, Matos HJ, Borges MA, Bordallo MAN. Ponto de corte do índice Homeostatic Model Assessment for Insulin Resistance (HOMA-IR) avaliado pela curva Receiver Operating Characteristic (ROC) na detecção de síndrome metabólica em crianças pré-púberes com excesso de peso. Arq Bras Endocrinol Metab. 2008;52(9):1466-73.

9. Penaforte FRO, Japur CC, Diez RW, Chiarello PG. Perfil antropométrico e bioquímico, apetite e consumo alimentar ad libitum em mulheres obesas com e sem resistência a insulina. Nutrire. 2013;38:98-98.

10. Santos CRB, Portella ES, Avila SS, Lanzillotti HS, Soares EA. Avaliação Nutricional de pacientes diabéticos tipo 2 . Rev Ciênc Méd. 2005;14(2):119-28.

11. Anunciação PC, Braga PG, Almeida OS, Lobo, LN, Pessoa, MC. Avaliação do conhecimento sobre alimentação antes e após intervenção nutricional entre diabéticos tipo 2. Rev Baiana Saúde Pública. 2012;36(4):986-1001.

12. Natali CM, Oliveira MCF, Alfenas RCG, Araújo RMA, Santana LFR, Cecon PR et al. Impactos dos estágios de mudança de comportamentos alimentares e de atividade física nos perfis bioquímico e antropométrico de portadores de Diabetes mellitus tipo 2. Nutrire: ver. Soc Bras Alim Nutr. 2012;37(3):322-34.

13. Bressan J, Hermsdorff HHM, Zulet MA, Martinez, JA. Impacto hormonal e inflamatório de diferentes composições dietéticas: ênfase em padróes alimentares e fatores dietéticos específicos. Arq Bras Endocrinol Metab. 2009;53(5):572-81.

14. SBC. Socidade Brasileira de Cardiologia. I Diretriz Brasileira de Diagnóstico e Tratamento da Síndrome Metabólica. Arq Bras Cardiol. 2005;84:3-28.

15. Internacional Expert Committee. The International Expert Committee report on the role of the $\mathrm{A} 1 \mathrm{C}$ assay in the diagnosis of diabetes. Diabetes Care. 2009;32:1327-34.

16. Matthews DR, Hosker JP, Rudenski AS, Naylor BA, Treacher DF, Turner RC. Homeostasis model assessment: insulin resistance and $\beta$-cell function from fasting plasma glucose and insulin concentrations in man. Diabetologia. 1985;28(7):412-9.

17. Geloneze B, Vasques ACJ, Stabe CFC, Pareja JC, Rosado LEFP de L, Queiroz EC de et al. HOMA1-IR and HOMA2-IR indexes in identifying insulin resistance and metabolic syndrome: Brazilian Metabolic Syndrome Study (BRAMS). Arq Bras Endocrinol Metab. 2009;53(2):281-87.

18. Physical status: the use and interpretation of anthropometry. Report of the WHO Expert Committee. World Health Organ Tech Rep Ser. 1995;854:1-452.

19. Lipschitz DA. Screening for nutritional status in the elderly. PrimCare. 1994;(21):55-67.

20. SBC. Socidade Brasileira de Cardiologia. I Diretriz Brasileira de Diagnóstico e Tratamento da Síndrome Metabólica. Arq Bras Cardiol. 2005;84:3-28.
21. Vasques ACJ, Rosado LEFPL, Rosado GP, Ribeiro RCL, Franceschini SCC, Geloneze B et al. Habilidade de indicadores antropométricos e de composição corporal em identificar a resistência à insulina. Arq Bras Endocrinol Metab. 2009;53(1):72-9.

22. Sociedade Brasileira de Cardiologia. VI Diretrizes Brasileiras de Hipertensão. Arq Bras Cardiol. 2010;95:I-III.

23. Ferreira IMC, Jorcelino SPN, Cabral JM. Tratamento da diabetes mellitus tipo 2 e comorbidades hepáticas. Relato de caso e revisão da literatura. Rev Bras Clin Med. 2013;11(2):183-93

24. Sotero H, Uezima CB, Guimarães EM, Stempniewski PF, Carvalho MB, Paiva CC, Netto AP. Impacto de um programa de educaçáo nutricional sobre perfil bioquímico e consumo alimentar de paciente idosa com diabetes mellitus tipo 2 e controle glicêmico precário: Estudo de caso. Nutrire. 38:145-45.

25. Galvão R, Plavnik FL, Ribeiro FF, Ajzen SA, Christofalo DMJ, Kohlmann JO. Efeitos de diferentes graus de sensibilidade à insulina na função endotelial de pacientes obesos. Arq Bras Cardiol. 2012;98(1):45-51.

26. Aguiar RS, Manini R. A fisiologia da obesidade: bases genéticas, ambientais e sua relação com o diabetes. Com Ciência. 2013;(145):0-0.

27. Machado SP, Rodrigues DGC, Viana KDAL, Sampaio HAC. Correlação entre o índice de massa corporal e indicadores antropométricos de obesidade abdominal em portadores de diabetes mellitus tipo 2. Rev Bras Promoç Saúde. 2012;25(4):512-20.

28. Faria AN, Zanella MT, Kohlman O, Ribeiro AB. Tratamento de Diabetes e Hipertensão no Paciente Obeso. Arq Bras Endocrinol Metab. 2002;46(2):137-42.

29. Martins DV, Araújo L; SBEM/SBN. Diabetes Mellitus: Tratamento de Hipertensão Arterial. Projeto diretrizes. 2004:1-8.

30. Gagliardi ART. Resistência À Insulina. Atheros. 2002;13(2):39-41.

31. Reaven, GM. Relationships Among Insulin Resistance, Type2 Diabetes, Essential Hypertension, and Cardiovascular Disease: Similarities and Differences. The Journal of Clinical Hypertension. 2011;13:238-43.

32. Santos-Filha EO, Melo IRC, Oliveira LMS, Souza MFC. Prevalência de excesso de peso, hipertensão e dislipidemia em pacientes diabéticos. Nutrire, 2013;38:143-43.

33. Xavier HT, Izar MC, Faria Neto JR, Assad MH, Rocha VZ, Sposito AC et al. V Diretriz Brasileira de Dislipidemias e Prevenção da Aterosclerose. Arq Bras Cardiol. 2013;101(4 Suppl 1):1-20.

34. Vasques ACJ, Rosado LEFPM, Rosado GP, et al. Indicadores do perfil lipídico plasmático relacionados à resistência à insulina. Rev Assoc Méd Bras. 2009;55(3):342-6.

35. Nogueira FilhoJ. Dislipidemia no Diabete Melito Tipo 2. In: Martinez TLR, Nogueira Filho J, Kencis Júnior L, Fernandes SC, Lopes AC. Dislipidemias: da teoria à prática. 1aed. São Paulo: Editora Atheneu. 2004:95-110. 
36. Carvalheira JBC, Saad MJA. Doenças associadas à resistência à insulina/hiperinsulinemia, não incluídas na síndrome metabólica. Arq Bras Endocrinol Metab. 2006;50(2):360-67.

37. Abreu EMRG. Hiperuricemia e a resistência à insulina, [Dissertação de Mestrado Saúde Pública]. Porto: Faculdade de Medicina da Universidade do Porto e Instituto de Ciências Biomédicas Abel Salazar; 2011.59p.

38. Juraschek SP, McAdams-Demarco M, Miller ER, Gelber AC, Maynard JW, Pankow JS et al. Temporal Relationship Between Uric Acid Concentration and Risk of Diabetes in a Community-based Study Population. Am J Epidemiol. 2014;179(6):684-91.

39. Oliveira PB, Franco LJ. Consumo de adoçantes e produtos dietéticos por indivíduos com diabetes melito tipo 2, atendidos pelo Sistema Único de Saúde em Ribeirão Preto, SP. Arq Bras Endocrinol Metab. 2010;54(5):455-62.

40. American Diabetes Association. Evidence-based nutrition principles and recommendations for the treatment and prevention of diabetes and related complications (Position Statement). Diabetes Care. 2002;25(Suppl.1):202-12.

41. Saito T, Pereira RB, Paixão MPCP. Avaliação do nível de conhecimento de portadores de diabetes mellitus sobre adoçantes. Demetra. 2013;8(1):39-51.

42. Duarte MJD, Oliveira TC. Conhecimento e forma de consumo de adoçantes e produtos dietéticos em indivíduos hipertensos e diabéticos do programa hiperdia de um PSF do município de Itabira, MG. Rev Digital de Nutrição. 2008;2(2).

Como citar este artigo:

Rodrigues CN, Cândido FG, Alfenas RCG, Hermsdorff HHM. Resistência à insulina e diabetes tipo 2: uma análise transversal em um programa de intervenção nutricional. Rev. Aten. Saúde. 2015;13(46):11-22 